\title{
Extremal Solutions and Instantaneous Complete Blow-up for Elliptic and Parabolic Problems
}

\author{
Xavier Cabré* \\ ICREA and Universitat Politècnica de Catalunya \\ Departament de Matemàtica Aplicada 1 \\ Diagonal 647. 08028 Barcelona. SPAIN. xavier.cabre@upc.edu
}

\section{Introduction}

This article is concerned with several semilinear elliptic problems of the form

$$
\left\{\begin{aligned}
-\Delta u & =g_{\lambda}(x, u) & & \text { in } \Omega \\
u & =0 & & \text { on } \partial \Omega,
\end{aligned}\right.
$$

that admit a minimal solution for each $\lambda \leq \lambda^{*}$, where $\lambda^{*}$ is a certain extremal parameter, and having no solution for every $\lambda>\lambda^{*}$. The minimal solution $u^{*}$

corresponding to $\lambda=\lambda^{*}$ is called the extremal solution. An example of this situation is given by Gelfand's problem

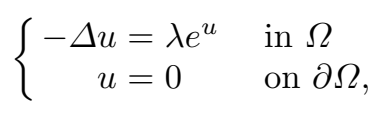

and more generally by

$$
\left\{\begin{aligned}
-\Delta u & =\lambda g(u) & & \text { in } \Omega \\
u & =0 & & \text { on } \partial \Omega,
\end{aligned}\right.
$$

whenever $g>0$ is increasing and convex on $\mathbb{R}$ and superlinear at $+\infty$ in the sense (7) stated below. Under these assumptions, the extremal parameter of (2) satisfies $0<\lambda^{*}<\infty$. Here and throughout the paper, $\Omega$ is a smooth bounded domain of $\mathbb{R}^{N}$.

For each $0 \leq \lambda<\lambda^{*}$, the minimal solution of (2) is classical. Their limit as $\lambda \uparrow \lambda^{*}$ is the extremal solution $u^{*}$ and it may be singular (i.e. unbounded) for some nonlinearities and domains. When $g(u)=e^{u}$, it is known that $u^{*} \in$ $L^{\infty}(\Omega)$ if $N \leq 9$ (for every $\Omega$ ), while $u^{*}(x)=\log \left(1 /|x|^{2}\right)$ if $N \geq 10$ and $\Omega=B_{1}$. Brezis and Vázquez [BV] raised the question of determining the

\footnotetext{
* Supported by grant BFM2002-04613-C03-01 from the Spanish Government
} 
regularity of $u^{*}$, depending on the dimension $N$, for general nonlinearities $g$ as above. Below we explain recent results of Nedev [N1, N2] and of the author and Capella [CCa2] on this regularity issue.

While nonexistence of classical solutions for $\lambda>\lambda^{*}$ follows by the definition of $\lambda^{*}$, Brezis, Cazenave, Martel and Ramiandrisoa [BCMR] proved that, in addition, no weak solution of (2) exists for $\lambda>\lambda^{*}$. Later, Brezis and Vázquez [BV] characterized singular extremal solutions of (2) by means of a stability condition. For some equations in dimension $N \geq 11$, they also show that an apparent "failure" of the Implicit Function Theorem occurs at $\left(u^{*}, \lambda^{*}\right)$, since the linearized problem at $u^{*}$ turns out to be formally invertible (for some problems) and, however, no weak solution exists for $\lambda>\lambda^{*}$.

This article is a survey of several results and methods from [BC, BCMR, BV, CCa2, CMa1, CMa2, Ma1, N1, N2, PV] concerning the previous issues, both in the elliptic and the parabolic settings. Special attention will be made on the regularity of the extremal solution $u^{*}$, as well as on how nonexistence develops for $\lambda>\lambda^{*}$. We will see that this last question is related to a phenomenon of complete blow-up of approximate solutions, and of instantaneous complete blow-up in the parabolic setting.

Sections 2 and 3 are devoted to existence, nonexistence, uniqueness, stability and boundedness of solutions of (2). We describe some results of [BCMR, BV, CCa2, CMa1, Ma1, N1, N2] about weak solutions of (2). We are particularly interested on the regularity of the extremal solution $u^{*}$ and on the linearized problem of (2) at the extremal solution and its connections with the Implicit Function Theorem.

Section 4 is concerned with $[\mathrm{BC}]$, where Brezis and the author make a detailed analysis of how nonexistence develops for $\lambda>\lambda^{*}$, and also of the apparent "failure" of the Implicit Function Theorem, for the simple model

$$
\left\{\begin{aligned}
-\Delta u & =\frac{u^{2}}{|x|^{2}}+\lambda & & \text { in } \Omega \\
u & =0 & & \text { on } \partial \Omega,
\end{aligned}\right.
$$

in all dimensions $N \geq 2$. Here $0 \in \Omega, u^{*} \equiv 0$ and $\lambda^{*}=0$. Indeed, it is proved in $[\mathrm{BC}]$ that, for every $\lambda>0$, (3) has no solution in a very weak sense. Moreover, approximate solutions of (3) blow-up everywhere in $\Omega$, i.e., there is complete blow-up in the sense established by Baras and Cohen [BaCo] for some parabolic problems.

In Section 5 we present related nonexistence and blow-up results obtained in $[\mathrm{BC}]$ for the parabolic version of (3). They extend, in particular, a result of Peral and Vázquez [PV]. Namely, the problem

$$
\left\{\begin{aligned}
u_{t}-\Delta u & =2(N-2) e^{u} & & \text { in }(0, T) \times B_{1} \\
u & =0 & & \text { on }(0, T) \times \partial B_{1} \\
u(0) & =u_{0} & & \text { on } B_{1},
\end{aligned}\right.
$$

with $u_{0} \geq \bar{u}:=\log \left(1 /|x|^{2}\right)$ and $u_{0} \not \equiv \bar{u}$, has no weak solution $u(t, x) \geq \bar{u}(x)$ even for small time: instantaneous complete blow-up occurs. To prove this 
result we will consider the equation satisfied by $u-\bar{u}$. It leads to the parabolic version of problem (3).

The potential $|x|^{-2}$ also appears when linearizing problem (4) at $\bar{u}$. Hence, the study of the linear problem $u_{t}-\Delta u=\lambda|x|^{-2} u$ is also of interest. In Section 6 we discuss the work of Baras and Goldstein $[\mathrm{BaG}]$ on this equation, as well as results of the author and Martel [CMa2] on linear parabolic equations with singular potentials:

$$
\left\{\begin{aligned}
u_{t}-\Delta u & =a(x) u & & \text { in }(0, T) \times \Omega \\
u & =0 & & \text { on }(0, T) \times \partial \Omega \\
u(0) & =u_{0} & & \text { on } \Omega,
\end{aligned}\right.
$$

with $a \geq 0$ and $u_{0} \geq 0$ in $L_{\text {loc }}^{1}(\Omega)$. We give conditions, based on the validity of a Hardy type inequality with weight $a(x)$, that ensure either the existence of global weak solutions or the instantaneous complete blow-up of approximate solutions of (5). Replacing $a(x)$ by $\lambda a(x)$ in (5), the extremal parameter $\lambda^{*}$ can be explicitly computed for some critical potentials $a(x)$.

Our work extends a result proved by Baras and Goldstein [BaG] in 1984 . Namely, if $a(x)=\lambda|x|^{-2}, 0 \in \Omega$ and $N \geq 3$, then $\lambda^{*}=(N-2)^{2} / 4$. More precisely, (5) has a global weak solution for every $u_{0} \in L^{2}(\Omega)$ and every $0 \leq \lambda \leq \lambda^{*}=(N-2)^{2} / 4$. Instead, if $\lambda>\lambda^{*}=(N-2)^{2} / 4$ then (5) has no positive weak solution for every $T>0$ and every $u_{0} \in L_{\text {loc }}^{1}(\Omega)$ with $u_{0} \geq 0$ and $u_{0} \not \equiv 0$.

The paper is organized as follows:

1. Introduction.

2. The extremal solution.

3. An apparent "failure" of the Implicit Function Theorem.

4. Nonlinear elliptic problems: very weak solutions.

5. Nonlinear parabolic problems.

6. Linear parabolic problems with singular potentials.

\section{The extremal solution}

We start considering the problem

$$
\left\{\begin{aligned}
-\Delta u & =\lambda g(u) & & \text { in } \Omega \\
u & =0 & & \text { on } \partial \Omega,
\end{aligned}\right.
$$

where $\lambda \geq 0$ is a parameter, and $\Omega$ is a smooth bounded domain of $\mathbb{R}^{N}$, $N \geq 2$. The nonlinearity $g$ is a $C^{1}$, increasing and convex function on $[0, \infty)$ such that

$$
g(0)>0 \text { and } \quad \lim _{u \rightarrow+\infty} \frac{g(u)}{u}=+\infty .
$$


Since $g>0$, we consider nonnegative solutions of $\left(6_{\lambda}\right)$. The cases $g(u)=e^{u}$ and $g(u)=(1+u)^{p}$, with $p>1$, are classical examples of such nonlinearities.

These reaction-diffusion problems appear in numerous models in physics, chemistry and biology. The case of an exponential reaction term $g(u)=e^{u}$ is a very simplified model in combustion theory. Since the work of Gelfand [G] in the sixties, problem $\left(6_{\lambda}\right)$ has been extensively studied.

\subsection{Minimal stable solutions}

It is well known that there exists a parameter $\lambda^{*}$ with $0<\lambda^{*}<\infty$, called the extremal parameter, such that if $0 \leq \lambda<\lambda^{*}$ then $\left(6_{\lambda}\right)$ has a minimal classical solution $u_{\lambda}$. On the other hand, if $\lambda>\lambda^{*}$ then $\left(6_{\lambda}\right)$ has no classical solution.

The set $\left\{u_{\lambda} ; 0 \leq \lambda<\lambda^{*}\right\}$ forms a branch of solutions increasing in $\lambda$. Moreover, every solution $u_{\lambda}$ is stable, in the sense that the first Dirichlet eigenvalue of the linearized problem at $u_{\lambda}$ is positive:

$$
\mu_{1}\left\{-\Delta-\lambda g^{\prime}\left(u_{\lambda}\right) ; \Omega\right\}>0 .
$$

In particular, for the quadratic form associated to the linearized problem we have

$$
Q_{u_{\lambda}}(\varphi):=\int_{\Omega}|\nabla \varphi|^{2}-\int_{\Omega} \lambda g^{\prime}\left(u_{\lambda}\right) \varphi^{2} \geq 0 \quad \forall \varphi \in H_{0}^{1}(\Omega) .
$$

This condition, that we call semi-stability, is equivalent to $\mu_{1} \geq 0$, where $\mu_{1}$ is the first eigenvalue of the linearized problem as above.

Recall that the first Dirichlet eigenvalue in $\Omega$ of a linear operator of the form $-\Delta-a(x)$ is defined by

$$
\mu_{1}\{-\Delta-a(x) ; \Omega\}=\inf _{0 \neq \varphi \in C_{c}^{\infty}(\Omega)} \frac{\int_{\Omega}|\nabla \varphi|^{2}-\int_{\Omega} a(x) \varphi^{2}}{\int_{\Omega} \varphi^{2}} .
$$

Later, we will also use this expression as definition of generalized first eigenvalue when $a \in L_{\text {loc }}^{1}(\Omega), a \geq 0$ a.e. and $a$ is singular. In this case, $\mu_{1}\{-\Delta-a(x) ; \Omega\}$ could be $-\infty$.

The existence of the branch $\left\{u_{\lambda} ; 0 \leq \lambda<\lambda^{*}\right\}$ can be proved using the Implicit Function Theorem (starting from $\lambda=0$ ). The solution $u_{\lambda}$ may also be obtained by the monotone iteration procedure, with $0<\lambda<\lambda^{*}$ fixed, starting from $u \equiv 0$ (note that $u \equiv 0$ is a strict subsolution of the problem). Finally, let us recall that the minimal solution $u_{\lambda}$ is smaller than every other solution or supersolution of $\left(6_{\lambda}\right)$. These results, among others concerning $\left(6_{\lambda}\right)$, are proved in $[\mathrm{CrR}]$ and $[\mathrm{MP}]$.

\subsection{Weak solutions}

The increasing limit of $u_{\lambda}$ as $\lambda \uparrow \lambda^{*}$ is called the extremal solution $u^{*}$. It is proved in [BCMR] that $u^{*}$ is a weak solution of $\left(6_{\lambda}\right)$ for $\lambda=\lambda^{*}$ in the following sense: 
Definition 1. We say that $u$ is a weak solution of $\left(6_{\lambda}\right)$ if

$$
u \in L^{1}(\Omega), \quad g(u) \delta \in L^{1}(\Omega), \quad \text { and } \quad-\int_{\Omega} u \Delta \zeta=\int_{\Omega} \lambda g(u) \zeta
$$

for all $\zeta \in C^{2}(\bar{\Omega})$ with $\zeta=0$ on $\partial \Omega$, where $\delta(x)=\operatorname{dist}(x, \partial \Omega)$ denotes the distance to the boundary of $\Omega$.

More generally, the definition of weak solution for problem (1) is the analogous one: we now assume $g_{\lambda}(x, u(x)) \delta(x) \in L^{1}(\Omega)$ and we replace $\lambda g(u) \zeta$ by $g_{\lambda}(x, u) \zeta$ in the above integral.

The nonexistence of classical solution for $\lambda>\lambda^{*}$ has been improved by Brezis, Cazenave, Martel and Ramiandrisoa [BCMR]:

Theorem 1 ([BCMR $])$. There is no weak solution of $\left(6_{\lambda}\right)$ for every $\lambda>\lambda^{*}$.

In case $g(u)=e^{u}$, a similar result had been obtained by Gallouët, Mignot and Puel [GMP].

The proof of Theorem 1 given in $[\mathrm{BCMR}]$ uses a new and interesting method. One assumes that $\left(6_{\lambda}\right)$ has a weak solution for some $\lambda>\lambda^{*}$. One then considers the equation satisfied by $\phi(u)$, where $\phi$ is a positive, bounded and concave function which is chosen appropriately depending on the nonlinearity $g$. It is possible to construct in this way a bounded supersolution (and hence a classical solution) of $\left(6_{(1-\varepsilon) \lambda}\right)$, for each $0<\varepsilon<1$. But this is a contradiction if $\varepsilon$ is small enough so that $(1-\varepsilon) \lambda>\lambda^{*}$.

This procedure could be called the "method of generalized truncations" $\phi(u)$ of $u$. It is also used in [BCMR] to study the global existence and the blow-up in finite time of solutions to the evolution problem $u_{t}-\Delta u=\lambda g(u)$, and in [Ma2] to prove results on complete blow-up of solutions. A refined version of the method is also used by Martel [Ma1] to show the following property of extremal solutions:

Theorem 2 ([Ma1]). $u^{*}$ is the unique weak solution of $\left(6_{\lambda^{*}}\right)$.

\subsection{Regularity of the extremal solution}

The extremal solution $u^{*}$ may be classical or singular depending on each problem. For instance, for Gelfand's problem we have:

Theorem 3 ([CrR, MP, JL]). Let $u^{*}$ be the extremal solution of $\left(6_{\lambda}\right)$.

(i) If $g(u)=e^{u}$ and $N \leq 9$, then $u^{*} \in L^{\infty}(\Omega)$ (i.e., $u^{*}$ is classical in all $\Omega$ ).

(ii) If $\Omega=B_{1}$ (the unit ball), $g(u)=e^{u}$ and $N \geq 10$, then $u^{*}=\log \left(1 /|x|^{2}\right)$ and $\lambda^{*}=2(N-2)$. 
There is an analogous result for $g(u)=(1+u)^{p}$. In this case, the explicit radial solution is given by $|x|^{-2 /(p-1)}-1$ and coincides with the extremal solution $u^{*}$ for $\Omega=B_{1}$ depending on the values of $N$ and $p$ (see [BV, CCa2] for more details).

Part (i) of Theorem 3 was proven by Crandall and Rabinowitz $[\mathrm{CrR}]$ and by Mignot and Puel [MP]. The proof uses the stability of the minimal solutions, as follows. One takes $\varphi=e^{\alpha u_{\lambda}}-1$ in the semi-stability condition (8). One then uses that $u_{\lambda}$ is a solution of the problem. This leads to an $L^{1}(\Omega)$ bound for $e^{\beta u_{\lambda}}$, uniform in $\lambda$, for some exponents $\beta$. That is, $-\Delta u_{\lambda}$ is uniformly bounded in $L^{\beta}(\Omega)$ and, hence, $u_{\lambda}$ is uniformly bounded in $W^{2, \beta}(\Omega)$. If $N \leq 9$, the exponent $\beta$ turns out to be bigger that $N / 2$ and, therefore, we have a uniform $L^{\infty}(\Omega)$ bound for $u_{\lambda}$.

Part (ii) of the theorem had been proved by Joseph and Lundgren [JL] in their exhaustive study of the radial case. More recently, Brezis and Vázquez [BV] have introduced a simple approach to this question, based on the following characterization of singular extremal solutions by their semi-stability property:

Theorem 4 ([BV]). Let $u \in H_{0}^{1}(\Omega), u \notin L^{\infty}(\Omega)$, be an unbounded weak solution of $\left(6_{\lambda}\right)$ for some $\lambda>0$. Then, the following are equivalent:

(a) The solution $u$ satisfies

$$
\int_{\Omega}|\nabla \varphi|^{2}-\int_{\Omega} \lambda g^{\prime}(u) \varphi^{2} \geq 0 \quad \forall \varphi \in H_{0}^{1}(\Omega) .
$$

(b) $\lambda=\lambda^{*}$ and $u=u^{*}$.

That (a) holds for $u^{*}$ follows immediately from the semi-stability (8) of minimal solutions, by letting $\lambda \uparrow \lambda^{*}$. The key idea behind the other implication of the theorem is that, for each $\lambda>0,\left(6_{\lambda}\right)$ has at most one semi-stable solution -a consequence of the convexity of $g$.

Following $[\mathrm{BV}]$, we can deduce part (ii) of Theorem 3 from Theorem 4 . Indeed, let $\Omega=B_{1}$ and $\bar{u}=\log \left(1 /|x|^{2}\right)$. A direct computation shows that this function is a solution of $\left(6_{\lambda}\right)$ for $\lambda=\bar{\lambda}=2(N-2)$. The linearized operator at $\bar{u}$ is given by

$$
L \varphi=-\Delta \varphi-2(N-2) e^{\bar{u}} \varphi=-\Delta \varphi-\frac{2(N-2)}{|x|^{2}} \varphi .
$$

If $N \geq 10$ then the first eigenvalue of $L$ in $B_{1}$ satisfies $\mu_{1}\left\{L ; B_{1}\right\} \geq 0$. This is a consequence of Hardy's inequality:

$$
\frac{(N-2)^{2}}{4} \int_{B_{1}} \frac{\varphi^{2}}{|x|^{2}} \leq \int_{B_{1}}|\nabla \varphi|^{2} \quad \forall \varphi \in H_{0}^{1}\left(B_{1}\right),
$$

and the fact that $(N-2)^{2} / 4 \geq 2(N-2)$ if $N \geq 10$. Applying Theorem 4 to $(\bar{u}, \bar{\lambda})$ we deduce part (ii) of Theorem 3 . 
Before proceeding we recall that, by scale invariance, Hardy's inequality also holds in every domain $\Omega \subset \mathbb{R}^{N}$ with $N \geq 3$. Namely,

$$
\frac{(N-2)^{2}}{4} \int_{\Omega} \frac{\varphi^{2}}{|x|^{2}} \leq \int_{\Omega}|\nabla \varphi|^{2} \quad \forall \varphi \in H_{0}^{1}(\Omega) .
$$

Moreover, if $0 \in \Omega$ then the optimal constant is $(N-2)^{2} / 4$ and it is not attained in $H_{0}^{1}(\Omega)$; see [BV].

Theorem 3 is a precise result on the boundedness of $u^{*}$ when $g(u)=e^{u}$. A related regularity result from $[\mathrm{BV}]$ states that if the nonlinearity satisfies in addition $\liminf _{u \rightarrow \infty} u g^{\prime}(u) / g(u)>1$, then $u^{*} \in H_{0}^{1}(\Omega)$ for all $\Omega$ and $N$. However, to establish regularity of $u^{*}$ without additional assumptions on $g-\mathrm{a}$ question raised by Brezis and Vázquez [BV]- is a much harder task. The best known results for general domains and nonlinearities as in the beginning of Section 2 are due to Nedev [N1, N2]:

Theorem $5([\mathbf{N 1}, \mathbf{N} 2])$. Let $u^{*}$ be the extremal solution of $\left(6_{\lambda}\right)$.

(i) If $N \leq 3$, then $u^{*} \in L^{\infty}(\Omega)$ (for every $\Omega$ ).

(ii) If $N \leq 5$, then $u^{*} \in H_{0}^{1}(\Omega)$ (for every $\Omega$ ).

(iii) For every dimension $N$, if $\Omega$ is strictly convex then $u^{*} \in H_{0}^{1}(\Omega)$.

The proof of this theorem uses a very refined version of the method of [CrR, MP] described above in relation to Theorem 3.

There are still many questions to be answered in the case of general $\Omega$ and $g$. For instance, it is not known if an extremal solution may be singular in dimensions $4 \leq N \leq 9$, for some domain and nonlinearity.

However, the radial case $\Omega=B_{1}$ has been recently settled by the author and Capella [CCa2]. The result establishes optimal regularity results for general $g$. To state it, we define exponents $q_{k}$ for $k \in\{0,1,2,3\}$ by

$$
\begin{cases}\frac{1}{q_{k}}=\frac{1}{2}-\frac{\sqrt{N-1}}{N}+\frac{k-2}{N} & \text { for } N \geq 10 \\ q_{k}=+\infty & \text { for } N \leq 9 .\end{cases}
$$

Note that all the exponents are well defined and satisfy $2<q_{k} \leq+\infty$. In the same way as Theorem 5, the following result holds for every nonlinearity $g$ satisfying the assumptions at the beginning of Section 2 .

Theorem 6 ([CCa2]). Let $\Omega=B_{1}$ and $u^{*}$ be the extremal solution of $\left(6_{\lambda}\right)$.

(i) If $N \leq 9$, then $u^{*} \in L^{\infty}\left(B_{1}\right)$.

(ii) If $N=10$, then $u^{*}(|x|) \leq C \log (1 /|x|)$ in $B_{1}$ for some constant $C$.

(iii) If $N \geq 11$, then

$$
u^{*}(|x|) \leq C|x|^{-N / 2+\sqrt{N-1}+2} \sqrt{\log (1 /|x|)} \quad \text { in } B_{1}
$$

for some constant $C$. In particular, $u^{*} \in L^{q}\left(B_{1}\right)$ for every $q<q_{0}$. Moreover, for every $N \geq 11$ there exists $p_{N}>1$ such that $u^{*} \notin L^{q_{0}}\left(B_{1}\right)$ when $g(u)=$ $(1+u)^{p_{N}}$. 
(iv) $u^{*} \in W^{k, q}\left(B_{1}\right)$ for every $k \in\{1,2,3\}$ and $q<q_{k}$. In particular, $u^{*} \in H^{3}\left(B_{1}\right)$ for every $N$. Moreover, for all $N \geq 10$ and $k \in\{1,2,3\}$,

$$
\left|\partial^{k} u^{*}(|x|)\right| \leq C r^{-N / 2+\sqrt{N-1}+2-k}(1+\sqrt{\log (1 /|x|)}) \quad \text { in } B_{1}
$$

for some constant $C$. Here $\partial^{k} u^{*}$ denotes the $k$-th derivative of the radial function $u^{*}$.

Theorem 3, which deals with $g(u)=e^{u}$, shows the optimality of Theorem 6(i)(ii), including the logarithmic pointwise bound of part (ii). The $L^{q}$ regularity stated in part (iii) $\left(q<q_{0}\right)$ is also optimal. This is shown considering $g(u)=(1+u)^{p_{N}}$ (for an explicit $\left.p_{N}\right)$, in which case $u^{*}(|x|)=$ $|x|^{-N / 2+\sqrt{N-1}+2}-1$. This function differs from the pointwise power bound (13) for the factor $\sqrt{\log (1 /|x|)}$. It is an open problem to know if this logarithmic factor in (13) can be removed. The exponents $q_{k}$ in the Sobolev estimates of part (iv) are optimal. This follows immediately from the optimality of $q_{0}$ and the fact that all $q_{k}$ are related by optimal Sobolev embeddings.

The proof of Theorem 6 was inspired by the proof of Simons theorem on the nonexistence of singular minimal cones in $\mathbb{R}^{N}$ for $N \leq 7$. The key idea is to take $\varphi=\left(r^{-\beta}-1\right) u_{r}$, where $r=|x|$, and compute $Q_{u^{*}}\left(\left(r^{-\beta}-1\right) u_{r}\right)$ in the semi-stability property (8) satisfied by $u^{*}$. The nonnegativeness of $Q_{u^{*}}$ leads to an $L^{2}$ bound for $u_{r} r^{-\alpha}$, with $\alpha$ depending on the dimension $N$. This is the key point in the proof. A similar method was employed in [CCa1] to study stability properties of radial solutions in all of $\mathbb{R}^{N}$.

Theorem 6 has been extended in $[\mathrm{CCaS}]$ to equations involving the $p$ Laplacian and having general semilinear reaction terms. Previously, Boccardo, Escobedo and Peral [BEP] had extended Theorem 3 on the exponential nonlinearity to the $p$-Laplacian case in general domains.

\section{An apparent "failure" of the Implicit Function Theorem}

\subsection{Hardy's inequality and the Implicit Function Theorem}

As a consequence of (8), we know that the extremal solution is semi-stable, i.e., it satisfies $\mu_{1}\left\{-\Delta-\lambda^{*} g^{\prime}\left(u^{*}\right) ; \Omega\right\} \geq 0$. If $u^{*}$ is classical then necessarily $\mu_{1}\left\{-\Delta-\lambda^{*} g^{\prime}\left(u^{*}\right) ; \Omega\right\}=0$. This is an immediate consequence of the Implicit Function Theorem and the nonexistence of classical solutions of $\left(6_{\lambda}\right)$ for each $\lambda>\lambda^{*}$.

Brezis and Vázquez [BV] point out that the property $\mu_{1}\left\{-\Delta-\lambda^{*} g^{\prime}\left(u^{*}\right) ; \Omega\right\}$ $=0$ fails for some problems in which the extremal solution $u^{*}$ is singular. They also show that $\mu_{1}\left\{-\Delta-\lambda g^{\prime}\left(u_{\lambda}\right) ; \Omega\right\}$ may decrease to a positive number as $\lambda \uparrow \lambda^{*}$. An example of this situation is given by

$$
\left\{\begin{array}{clrl}
-\Delta u & =\lambda e^{u} & & \text { in } B_{1} \\
u & =0 & & \text { on } \partial B_{1},
\end{array}\right.
$$


in every dimension $N \geq 11$. Indeed, we know that for this problem $u^{*}=$ $\log \left(1 /|x|^{2}\right)$ and $\lambda^{*}=2(N-2)$. The linearized operator at $u^{*}$ is given by $L=-\Delta-2(N-2)|x|^{-2}$, which has positive first eigenvalue in $B_{1}$ if $N \geq 11$, by Hardy's inequality (11).

We conclude that, for $N \geq 11$, the linearized operator of (14) at $u^{*}$ is bijective, for example between $H_{0}^{1}\left(B_{1}\right)$ and $H^{-1}\left(B_{1}\right)$. However, the Implicit Function Theorem can not be applied to problem (14) at $\left(u^{*}, \lambda^{*}\right)$, since there are no weak solutions of (14) for $\lambda>\lambda^{*}$, by Theorem 1. Similarly, the Inverse Function Theorem can not be applied neither, since it is proved in [BCMR] that, for each $\lambda>0$, problem $-\Delta u=2(N-2) e^{u}+\lambda$ in $B_{1}, u=0$ on $\partial B_{1}$, admits no weak solution if $N \geq 10$.

This apparent contradiction is explained by the absence of appropriate functional spaces in which one could apply the Implicit or Inverse Function Theorems.

\subsection{Weak eigenfunctions}

Motivated by this apparent "failure" of the Implicit Function Theorem, the author and Martel [CMa1] have shown that the linearized problem of $\left(6_{\lambda^{*}}\right)$ at $u^{*}$ always admits a positive weak eigenfunction belonging to $L^{1}(\Omega)$ with eigenvalue 0 , even for the problems in which this operator has positive first eigenvalue in $H_{0}^{1}(\Omega)$. The fact that 0 is a weak eigenvalue is one explanation of the apparent "failure" of the Implicit Function Theorem at $u^{*}$.

Theorem 7 ([CMa1]). There exists a function $\varphi>0$ in $\Omega$ such that

$$
\left\{\begin{aligned}
-\Delta \varphi & =\lambda^{*} g^{\prime}\left(u^{*}\right) \varphi & & \text { in } \Omega \\
\varphi & =0 & & \text { on } \partial \Omega,
\end{aligned}\right.
$$

in the weak sense of Definition 1, i.e.,

$$
\varphi \in L^{1}(\Omega), \quad g^{\prime}\left(u^{*}\right) \varphi \delta \in L^{1}(\Omega), \quad \text { and } \quad-\int_{\Omega} \varphi \Delta \zeta=\int_{\Omega} \lambda^{*} g^{\prime}\left(u^{*}\right) \varphi \zeta
$$

for all $\zeta \in C^{2}(\bar{\Omega})$ with $\zeta=0$ on $\partial \Omega$. We recall that $\delta(x)=\operatorname{dist}(x, \partial \Omega)$. Moreover, $\varphi \in L^{q}(\Omega)$ for every $1 \leq q<N /(N-2)$.

We also prove the existence of a positive weak eigenfunction of the operator $-\Delta-\lambda^{*} g^{\prime}\left(u^{*}\right)$ with eigenvalue $\mu$, for every $\mu$ such that

$$
0 \leq \mu \leq \lim _{\lambda \uparrow \lambda^{*}} \mu_{1}\left\{-\Delta-\lambda g^{\prime}\left(u_{\lambda}\right) ; \Omega\right\} .
$$

Hence, there is a phenomenon of continuum spectrum if this limit is positive and, in particular, if $\mu_{1}\left\{-\Delta-\lambda^{*} g^{\prime}\left(u^{*}\right) ; \Omega\right\}>0$.

To prove Theorem 7, we approximate $g$ by an increasing sequence of convex functions $g_{n}$ for which the extremal solution $u_{n}^{*}$ is classical. For this, it 
is enough to take $g_{n}$ with subcritical growth, by standard regularity theory. We know that $\mu_{1}\left\{-\Delta-\lambda_{n}^{*} g_{n}^{\prime}\left(u_{n}^{*}\right) ; \Omega\right\}=0$, where $\lambda_{n}^{*}$ is the extremal parameter corresponding to $g_{n}$. We consider the first eigenfunction $\varphi_{n}$, which has eigenvalue 0 , of $-\Delta-\lambda_{n}^{*} g_{n}^{\prime}\left(u_{n}^{*}\right)$, normalized so that $\left\|\varphi_{n}\right\|_{L^{1}(\Omega)}=1$. We show that, up to subsequences, $u_{n}^{*}$ and $\varphi_{n}$ converge in $L^{r}(\Omega)$, for every $1 \leq r<N /(N-1)$, respectively to $u^{*}$ and $\varphi$, with $\varphi>0$ a weak solution of (15) as in the theorem. The key step of passing to the limit as $n \rightarrow \infty$ in the approximate equations is accomplished through an equi-integrability result. A similar method was employed by Baras and Cohen $[\mathrm{BaCo}]$ for some nonlinear parabolic problems.

Next, we make a detailed study of all weak eigenfunctions of (15) whenever $\Omega=B_{1}$ is the unit ball, $g(u)=e^{u}$ or $g(u)=(1+u)^{p}$, with $p>1$, and $u^{*}$ is singular. In these cases, we know that $u^{*}$ can be written explicitly, and that the corresponding linearized operator is $-\Delta-c|x|^{-2}$, with $0<c \leq(N-2)^{2} / 4$. We express all weak eigenfunctions and eigenvalues of

$$
\left\{\begin{aligned}
-\Delta \varphi & =\frac{c}{|x|^{2}} \varphi+\mu \varphi & & \text { in } B_{1} \\
\varphi & =0 & & \text { on } \partial B_{1},
\end{aligned}\right.
$$

in terms of harmonic polynomials and Bessel functions. Looking at which of these eigenfunctions belong to $H_{0}^{1}\left(B_{1}\right)$, we obtain the following improved Hardy inequality due to Brezis and Vázquez [BV]:

Theorem 8 ([BV]). If $N \geq 2$ then

$$
\frac{(N-2)^{2}}{4} \int_{\Omega} \frac{\varphi^{2}}{|x|^{2}}+H_{2}\left(\frac{\omega_{N}}{|\Omega|}\right)^{2 / N} \int_{\Omega} \varphi^{2} \leq \int_{\Omega}|\nabla \varphi|^{2} \quad \forall \varphi \in H_{0}^{1}(\Omega),
$$

where $\mathrm{H}_{2}>0$ is the first eigenvalue of the Laplacian in the unit ball of $\mathbb{R}^{2}$, and $\omega_{N}$ is the measure of the unit ball of $\mathbb{R}^{N}$.

\section{Nonlinear elliptic problems: very weak solutions}

Brezis and the author [BC] have studied in detail how nonexistence develops for $\lambda>\lambda^{*}$, and also the phenomenon related to the Implicit Function Theorem, for the following model problem. Let $\Omega$ be a smooth bounded domain of $\mathbb{R}^{N}, N \geq 2$, such that $0 \in \Omega$. For $\lambda \in \mathbb{R}$, consider the problem

$$
\left\{\begin{aligned}
-\Delta u & =\frac{u^{2}}{|x|^{2}}+\lambda & & \text { in } \Omega \\
u & =0 & & \text { on } \partial \Omega .
\end{aligned}\right.
$$

A formal analysis suggests the existence of a small solution of (16) for $\lambda$ small, since the linearized operator at the solution $(u, \lambda)=(0,0)$ is $-\Delta$, which 
is bijective. However, we show that for every $\lambda>0$ (no matter how small), (16) has no solution in various weak senses. On the other hand, the results of [BS] show that (16) has a minimal negative solution for every constant $\lambda<0$. Hence, for this problem we have $u^{*}=0$ and $\lambda^{*}=0$.

\subsection{Non-existence}

The nonexistence of generalized solutions of (16) for $\lambda>0$ follows from the following result:

Theorem 9 ([BC]). If $0 \in \Omega, u \in L_{\text {loc }}^{2}(\Omega \backslash\{0\}), u \geq 0$ a.e., and

$$
-|x|^{2} \Delta u \geq u^{2} \quad \text { in } \mathcal{D}^{\prime}(\Omega \backslash\{0\}),
$$

then $u \equiv 0$. Here $\mathcal{D}^{\prime}(\Omega \backslash\{0\})$ denotes the space of distributions in $\Omega \backslash\{0\}$.

Theorem 9 is proved using appropriate powers of test functions -a method due to Baras and Pierre $[\mathrm{BaP}]$.

An immediate consequence of the theorem is the nonexistence of generalized solutions (so called very weak solutions in $[\mathrm{BC}]$ ) for the following problem:

Theorem 10 ([BC]). If $0 \in \Omega, f \geq 0$ a.e., and $f \not \equiv 0$, then

$$
\left\{\begin{aligned}
-\Delta u & =\frac{u^{2}}{|x|^{2}}+f(x) & & \text { in } \Omega \\
u & =0 & & \text { on } \partial \Omega,
\end{aligned}\right.
$$

has no solution $u \in L_{\mathrm{loc}}^{2}(\Omega \backslash\{0\})$ in the sense of distributions $\mathcal{D}^{\prime}(\Omega \backslash\{0\})$.

Note that, in the previous results, the test functions vanish in a neighborhood of 0 , and no assumption is made on the behavior of $u$ near 0 . In particular, these results imply nonexistence of weak solutions in the sense of Definition 1 in Section 2.

A second corollary of Theorem 9 is that $u \equiv 0$ is the only solution of (16) when $\lambda=0$ (compare this with the uniqueness property of $u^{*}$ stated in Theorem 2).

A last consequence of Theorem 9 is the nonexistence of local solutions (i.e., nonexistence in every neighborhood of 0 , without imposing any boundary condition) for a very simple nonlinear equation:

Theorem 11 ([BC]). If $0 \in \Omega$ and $\lambda>0$, then there is no function $u \in$ $L_{\text {loc }}^{2}(\Omega \backslash\{0\})$ such that

$$
-|x|^{2} \Delta u=u^{2}+\lambda \quad \text { in } \mathcal{D}^{\prime}(\Omega \backslash\{0\}) .
$$




\subsection{Complete blow-up}

Next, we study a natural approximation technique for problem (16). Consider, for example, the equation

$$
\left\{\begin{aligned}
-\Delta u & =\frac{\min \left\{u^{2}, n\right\}}{|x|^{2}+(1 / n)}+\lambda & & \text { in } \Omega \\
u & =0 & & \text { on } \partial \Omega
\end{aligned}\right.
$$

with $\lambda>0$. For every $n$, there exists a minimal solution $u_{n}$. We then have:

Theorem 12 ([BC]). If $\lambda>0$ then

$$
\frac{u_{n}(x)}{\delta(x)} \longrightarrow+\infty \quad \text { uniformly in } x \in \Omega \text {, as } n \rightarrow \infty \text {. }
$$

Hence, approximate solutions blow-up everywhere in $\Omega$, i.e., there is complete blow-up. This confirms that there is no reasonable notion of weak solution for (16) when $\lambda>0$.

The proof of Theorem 12 uses the nonexistence result for (16), the monotonicity property $u_{n} \leq u_{n+1}$, as well as an appropriate lower bound for the Green's function of the Laplacian in $\Omega$.

\subsection{More general problems}

The previous nonexistence and blow-up results are extended in $[\mathrm{BC}]$ to the problem

$$
\left\{\begin{aligned}
-\Delta u & =a(x) g(u)+f(x) & & \text { in } \Omega \\
u & =0 & & \text { on } \partial \Omega,
\end{aligned}\right.
$$

assuming only $g \geq 0$ on $\mathbb{R}, g$ is continuous and increasing on $[0, \infty)$,

$$
\int^{\infty} \frac{d s}{g(s)}<\infty
$$

with $a \in L_{\text {loc }}^{1}(\Omega), a \geq 0$,

$$
\int_{B_{\eta}(0)} \frac{a(x)}{|x|^{N-2}} d x=\infty
$$

for some $\eta>0$ small enough, and $f \in L_{\mathrm{loc}}^{1}(\Omega), f \geq 0$ and $f \not \equiv 0$.

For this problem, we prove nonexistence of solutions in the weak sense of Section 2, using a variant of the method of generalized truncations. Note that here we do not assume $g$ to be convex. 


\subsection{The existence criterium of Kalton and Verbitsky}

We now consider the previous problem in the case of power nonlinearities $g$, that is

$$
\left\{\begin{aligned}
-\Delta u & =a(x) u^{p}+f(x) & & \text { in } \Omega \\
u & =0 & & \text { on } \partial \Omega,
\end{aligned}\right.
$$

with $p>1, a$ and $f$ in $L_{\text {loc }}^{1}(\Omega), a \geq 0, a \not \equiv 0, f \geq 0$ and $f \not \equiv 0$ in $\Omega$.

Kalton and Verbitsky [KV] have found an interesting necessary condition for the existence of a weak solution of (17):

Theorem 13 ([KV]; see also [BC]). If (17) has a weak solution $u \geq 0$ (in the sense of Definition 1), then $a G(f)^{p} \delta \in L^{1}(\Omega)$ and

$$
\frac{G\left(a G(f)^{p}\right)}{G(f)} \leq \frac{1}{p-1} \quad \text { in } \Omega .
$$

Here $G=(-\Delta)^{-1}$ with zero Dirichlet boundary condition.

The explicit constant $1 /(p-1)$ was found in $[\mathrm{BC}]$. Note that Theorem 13 easily implies some of the previous nonexistence results. For instance, it gives Theorem 10 (for weak solutions instead of very weak solutions) whenever $f \in L^{\infty}(\Omega)$, since in this case $G(f) \sim \delta$.

The interest of Theorem 13 also lies in the fact that no assumption is made on the set of singularities of the potential $a$. In particular, $a$ could be singular near some parts of $\partial \Omega$. This is in contrast with previous problems considered in this paper, where $a$ had an isolated singularity in an interior point.

In $[\mathrm{BC}]$ we give a simple proof of condition (18) using a refinement of the method of generalized truncations described in Section 2. More precisely, we consider the equation satisfied by $v \psi(u / v)$, where $u$ is a weak solution of (17), $v=G(f)$ and $\psi$ is a bounded concave function appropriately chosen.

Next, we replace $f(x)$ by $\lambda f(x)$ in (17) and we study the problem of existence of solution depending on the value of $\lambda$. We then have:

Theorem 14 ([BC]). Assume that

$$
\frac{G\left(a G(f)^{p}\right)}{G(f)} \in L^{\infty}(\Omega) .
$$

For $\lambda \geq 0$, consider the problem

$$
\left\{\begin{aligned}
-\Delta u & =a(x) u^{p}+\lambda f(x) & & \text { in } \Omega \\
u & =0 & & \text { on } \partial \Omega .
\end{aligned}\right.
$$

Then there exists $\lambda^{*} \in(0, \infty)$ such that:

(i) If $0<\lambda<\lambda^{*}$, then (19) has a weak solution $u_{\lambda}$ satisfying

$$
\lambda \leq \frac{u_{\lambda}}{G(f)} \leq C(\lambda) \quad \text { in } \Omega
$$


for some constant $C(\lambda)$ depending on $\lambda$.

(ii) If $\lambda=\lambda^{*}$, then (19) has a weak solution.

(iii) If $\lambda>\lambda^{*}$, then (19) has no weak solution.

Moreover,

$$
\left(\frac{p-1}{p}\right)^{p} \frac{1}{p-1} \leq\left(\lambda^{*}\right)^{p-1}\left\|\frac{G\left(a G(f)^{p}\right)}{G(f)}\right\|_{L^{\infty}(\Omega)} \leq \frac{1}{p-1} .
$$

\section{Nonlinear Parabolic Problems}

The paper $[\mathrm{BC}]$ also contains parabolic versions of Theorems 9, 10 and 12 for very weak solutions of the equation

$$
\left\{\begin{aligned}
v_{t}-\Delta v & =\frac{v^{2}}{|x|^{2}} & & \text { in }(0, T) \times \Omega \\
v & =0 & & \text { on }(0, T) \times \partial \Omega \\
v(0) & =v_{0} & & \text { on } \Omega .
\end{aligned}\right.
$$

We show that for every initial condition $v_{0} \in L^{\infty}(\Omega), v_{0} \geq 0, v_{0} \not \equiv 0$, approximate solutions $v_{n}$ of (20) blow-up everywhere in $(0, T) \times \Omega$ (complete blow-up) and that this happens for all $T>0$ (instantaneous blow-up). More precisely, for all $0<\tau<T$, we have

$$
\frac{v_{n}(t, x)}{\delta(x)} \longrightarrow+\infty \quad \text { uniformly in }(\tau, T) \times \Omega \text {, as } n \rightarrow \infty \text {. }
$$

This extends the following result of Peral and Vázquez [PV]:

Theorem $15([\mathbf{P V}])$. Let $N \geq 3$. For every $u_{0} \geq \bar{u}:=\log \left(1 /|x|^{2}\right), u_{0} \not \equiv \bar{u}$, and for every $T>0$, the problem

$$
\left\{\begin{aligned}
u_{t}-\Delta u & =2(N-2) e^{u} & & \text { in }(0, T) \times B_{1} \\
u & =0 & & \text { on }(0, T) \times \partial B_{1} \\
u(0) & =u_{0} & & \text { on } B_{1}
\end{aligned}\right.
$$

has no weak solution $u$ such that $u(t, x) \geq \bar{u}(x)$ in $(0, T) \times B_{1}$.

In $[\mathrm{BC}]$ we give a simple proof of this theorem by applying the nonexistence results for (20) to $v:=u-\bar{u} \geq 0$, with $u$ as in Theorem 15. Indeed, it is easy to check that $v$ satisfies $v_{t}-\Delta v \geq(N-2)|x|^{-2} v^{2}$. We obtain nonexistence of solution of $(21)$ in the distributional sense $\mathcal{D}^{\prime}\left((0, T) \times\left(B_{1} \backslash\{0\}\right)\right)$. The assumptions on $u_{0}$ and $u$ in Theorem 15 have been improved and studied in detail by Vázquez [V]. 


\section{Linear parabolic problems with singular potentials}

We consider the linear heat equation with potential

$$
\left\{\begin{aligned}
u_{t}-\Delta u & =a(x) u & & \text { in }(0, T) \times \Omega \\
u & =0 & & \text { on }(0, T) \times \partial \Omega \\
u(0) & =u_{0} & & \text { on } \Omega,
\end{aligned}\right.
$$

where $\Omega$ is a smooth bounded domain of $\mathbb{R}^{N}$. We assume that $a \in L_{\text {loc }}^{1}(\Omega)$, $u_{0} \in L_{\text {loc }}^{1}(\Omega)$, and that $a \geq 0$ and $u_{0} \geq 0$ a.e. in $\Omega$. Note that $a$ is time independent. We only consider nonnegative solutions of (22).

Note that problem $(22)$ with $a(x)=2(N-2)|x|^{-2}$ is the linearization of $(21)$ at the stationary solution $\bar{u}$. In 1984, Baras and Goldstein [BaG] considered equation (22) with $a(x)=\lambda|x|^{-2}, 0 \in \Omega \subset \mathbb{R}^{N}$ and $N \geq 3$. For this problem they prove that if $0 \leq \lambda \leq(N-2)^{2} / 4=$ : $\lambda^{*}$ then there exists a global weak solution of $(22)$ for every $u_{0} \in L^{2}(\Omega)$. Instead, if $\lambda>$ $(N-2)^{2} / 4=\lambda^{*}$ then, for each $T>0$ and each $u_{0} \in L_{\text {loc }}^{1}(\Omega)$ with $u_{0} \geq 0$ and $u_{0} \not \equiv 0,(22)$ has no positive weak solution; moreover, in this case there is instantaneous complete blow-up of approximate solutions of (22). Their proof of nonexistence uses Moser's iteration technique, as well as a weighted Sobolev inequality.

The critical constant $\lambda^{*}=(N-2)^{2} / 4$ is related to Hardy's inequality (11). In [CMa2], the author and Martel consider the case of general potentials $a(x)$, and establish relations between the existence of solutions and the validity of a Hardy type inequality with weight $a(x)$. In particular, a new and simple proof of the nonexistence result of Baras and Goldstein is given.

In order to state the precise results, let $\delta(x)=\operatorname{dist}(x, \partial \Omega)$ for $x \in \Omega$, and let $L_{\delta}^{1}(\Omega)=L^{1}(\Omega, \delta(x) d x)$. For $0<T \leq \infty$ and $u_{0} \in L_{\delta}^{1}(\Omega)$, we say that $u \geq 0$ is a weak solution of (22) if, for each $0<S<T$, we have that $u \in L^{1}((0, S) \times \Omega), a u \delta \in L^{1}((0, S) \times \Omega)$, and

$$
\int_{0}^{S} \int_{\Omega} u\left(-\zeta_{t}-\Delta \zeta\right)-\int_{\Omega} u_{0} \zeta(0)=\int_{0}^{S} \int_{\Omega} a u \zeta
$$

for all $\zeta \in C^{2}([0, S] \times \bar{\Omega})$ with $\zeta(S) \equiv 0$ on $\Omega$ and $\zeta=0$ on $[0, S] \times \partial \Omega$. If $T=\infty$, we say that $u$ is a global weak solution.

Recall the definition of generalized first eigenvalue $\mu_{1}\{-\Delta-a(x) ; \Omega\}$ given by (9). The following result states that $\mu_{1}\{-\Delta-a(x) ; \Omega\}>-\infty$ is a necessary and sufficient condition for the existence of global weak solutions of (22) with (at most) exponential growth.

\section{Theorem 16 ([CMa2]).}

(i) Suppose that, for some $u_{0} \in L_{\delta}^{1}(\Omega)$ and some constants $C$ and $M$, there exists a global weak solution $u \geq 0$ of $(22)$ such that $\|u(t) \delta\|_{L^{1}(\Omega)} \leq C e^{M t}$ for all $t \geq 0$. Then $\mu_{1}\{-\Delta-a(x) ; \Omega\}>-\infty$. 
(ii) Suppose that $\mu_{1}\{-\Delta-a(x) ; \Omega\}>-\infty$. Then, for each $u_{0} \in L^{2}(\Omega)$ with $u_{0} \geq 0$, there exists a global weak solution $u \in C\left([0, \infty) ; L^{2}(\Omega)\right)$ of $(22)$ such that

$$
\|u(t)\|_{L^{2}(\Omega)} \leq\left\|u_{0}\right\|_{L^{2}(\Omega)} e^{-\mu_{1} t} \quad \text { for all } t \geq 0,
$$

where $\mu_{1}=\mu_{1}\{-\Delta-a(x) ; \Omega\}$.

The next result states that condition $\mu_{1}\{-\Delta-a(x) ; \Omega\}>-\infty$ is "almost necessary" for the local existence of weak solutions. More precisely, we have:

Theorem 17 ([CMa2]). Suppose that $\mu_{1}\{-\Delta-(1-\varepsilon) a(x) ; \Omega\}=-\infty$ for some constant $\varepsilon>0$. Then, for every $T>0$ and every $u_{0} \in L_{\delta}^{1}(\Omega)$ with $u_{0} \geq 0$ and $u_{0} \not \equiv 0$, there is no weak solution $u \geq 0$ of (22). Moreover, there is instantaneous complete blow-up for (22), in the following sense:

For every $n \geq 1$, set $a_{n}(x)=\min (a(x), n), u_{0 n}(x)=\min \left(u_{0}(x), n\right)$, and let $u_{n}$ be the unique global solution of (22) with a and $u_{0}$ replaced, respectively, by $a_{n}$ and $u_{0 n}$. Then, for all $0<\tau<T$,

$$
\frac{u_{n}(t, x)}{\delta(x)} \longrightarrow+\infty \quad \text { uniformly in }(\tau, T) \times \Omega \text {, as } n \rightarrow \infty \text {. }
$$

The following inequality is the key ingredient in the proof of the necessary conditions in these theorems. Suppose that (22) has a positive local solution, and let $\underline{u}$ be the minimal solution (i.e., the one obtained as increasing limit of approximate solutions). Then, for all $0<t_{1}<t_{2}<T$, we have

$$
\int_{\Omega} a(x) \varphi^{2}-\int_{\Omega}|\nabla \varphi|^{2} \leq \frac{1}{t_{2}-t_{1}} \int_{\Omega} \log \left(\frac{\underline{u}\left(t_{2}\right)}{\underline{u}\left(t_{1}\right)}\right) \varphi^{2} \quad \forall \varphi \in C_{c}^{\infty}(\Omega) .
$$

This inequality is proved multiplying the approximate problems of (22) by $\varphi^{2} / u_{n}$, then integrating by parts in space, integrating in time, and finally letting $n \rightarrow \infty$.

We apply the previous theorems to some specific equations with "critical" potentials. First, our method provides a new and quite elementary proof of the result of Baras and Goldstein $[\mathrm{BaG}]$ mentioned above. Namely, suppose that $0 \in \Omega \subset \mathbb{R}^{N}, N \geq 3$, and

$$
a(x)=\lambda|x|^{-2} .
$$

Let $\lambda^{*}=(N-2)^{2} / 4$. For this potential, [BaG] establishes the following:

(a) If $0 \leq \lambda \leq \lambda^{*}$, then (22) has a global weak solution for every $u_{0} \in$ $L^{2}(\Omega), u_{0} \geq 0$.

(b) If $\lambda>\lambda^{*}$, then (22) has no positive weak solution for every $T>0$ and every $u_{0} \in L_{\delta}^{1}(\Omega)$ with $u_{0} \geq 0$ and $u_{0} \not \equiv 0$. Moreover, there is instantaneous complete blow-up of approximate solutions. 
We obtain (a) and (b) through direct applications of Theorem 16(ii) and Theorem 17, respectively. Here the essential role is played by (11), i.e., Hardy's inequality with best constant.

In case $0 \leq \lambda \leq \lambda^{*}=(N-2)^{2} / 4$, we also obtain that solutions decay exponentially. More precisely, we have

$$
\|u(t)\|_{L^{2}(\Omega)} \leq\left\|u_{0}\right\|_{L^{2}(\Omega)} e^{-\mu t} \quad \text { for all } t \geq 0,
$$

where $\mu=H_{2}\left(\omega_{N} /|\Omega|\right)^{2 / N}>0$. This is a consequence of estimate (23) and the improved Hardy inequality (Theorem 8). New improved Hardy inequalities and a detailed description of solutions of (22) when $a(x)=\lambda|x|^{-2}$ and $0 \leq$ $\lambda \leq(N-2)^{2} / 4$ have been obtained by Vázquez and Zuazua [VZ]. In [GP], García Azorero and Peral have extended the results of Baras and Goldstein to the $p$-Laplace operator.

A second application of Theorems 16 and 17 is the case when $\Omega \subset \mathbb{R}^{N}$ is of class $C^{2}, N \geq 2$, and

$$
a(x)=\lambda \delta(x)^{-2} .
$$

Here $\lambda \geq 0$ is a constant and $\delta(x)=\operatorname{dist}(x, \partial \Omega)$. For this problem, we obtain statements (a) and (b) above with $\lambda^{*}=1 / 4$. Here we use some Hardy type inequalities proved in $[\mathrm{BM}]$ and $[\mathrm{D}]$.

Finally, recall that the results for the potential $\lambda|x|^{-2}$ required $N \geq 3$. In dimension $N=2$, we prove that the potential $a(x)=\lambda|x|^{-2}(1-\log |x|)^{-2}$ is "critical", in the sense that the dichotomy (a)-(b) occurs with $\lambda^{*}=1 / 4$ for all domains $\Omega$ with $0 \in \Omega \subset B_{1}(0) \subset \mathbb{R}^{2}$.

\section{References}

[BaCo] Baras, P., Cohen, L.: Complete blow-up after $T_{\max }$ for the solution of a semilinear heat equation. J. Funct. Anal., 71, 142-174 (1987)

[BaG] Baras, P., Goldstein, J.A.: The heat equation with a singular potential. Trans. Amer. Math. Soc., 284, 121-139 (1984)

[BaP] Baras, P., Pierre, M.: Singularités éliminables pour des équations semilinéaires. Ann. Inst. Fourier, 34, 185-206 (1984)

[BEP] Boccardo, L., Escobedo, M., Peral, I.: A Dirichlet problem involving critical exponents. Nonlinear Anal., Theory, Meth. \& Appl., 24, 1639-1648 (1995)

[BC] Brezis, H., Cabré, X.: Some simple nonlinear PDE's without solutions. Boll. Unione Mat. Ital., 1-B, 223-262 (1998)

[BCMR] Brezis, H., Cazenave, T., Martel, Y., Ramiandrisoa, A.: Blow up for $u_{t}-$ $\Delta u=g(u)$ revisited. Ad. Diff. Eq., 1, 73-90 (1996)

[BM] Brezis, H., Marcus M.: Hardy's inequalities revisited. Ann. Scuola Norm. Sup. Pisa Cl. Sci., 25, 217-237 (1997)

[BS] Brezis, H., Strauss, W.A.: Semi-linear second-order elliptic equations in $L^{1}$. J. Math. Soc. Japan, 25, 565-590 (1973)

[BV] Brezis, H., Vázquez, J.L.: Blow-up solutions of some nonlinear elliptic problems. Rev. Mat. Univ. Compl. Madrid, 10, 443-469 (1997) 
[CCa1] Cabré, X., Capella, A.: On the stability of radial solutions of semilinear elliptic equations in all of $\mathbb{R}^{n}$. C. R. Math. Acad. Sci. Paris, 338, 769-774 (2004)

[CCa2] Cabré, X., Capella, A.: Regularity of radial minimizers and extremal solutions of semilinear elliptic equations. Preprint, 2005

[CCaS] Cabré, X., Capella, A., Sanchón, M.: Regularity of radial minimizers and semi-stable solutions of semilinear problems involving the $p$-Laplacian. Preprint, 2005

[CMa1] Cabré, X., Martel, Y.: Weak eigenfunctions for the linearization of extremal elliptic problems. J. Funct. Anal., 156, 30-56 (1998)

[CMa2] Cabré, X., Martel, Y.: Existence versus instantaneous blowup for linear heat equations with singular potentials. C. R. Acad. Sci. Paris Sér. I Math., 329, 973-978 (1999)

[CrR] Crandall, M.G., Rabinowitz, P.H.: Some continuation and variational methods for positive solutions of nonlinear elliptic eigenvalue problems. Arch. Rat. Mech. Anal., 58, 207-218 (1975)

[D] Davies, E.B.: The Hardy constant. Quart. J. Math. Oxford, 46, 417-431 (1995)

[GMP] Gallouët, T., Mignot, F., Puel, J.-P.: Quelques résultats sur le problème $-\Delta u=\lambda e^{u}$. C. R. Acad. Sci. Paris, 307, Série I, 289-292 (1988)

[GP] García Azorero, J., Peral, I.: Hardy inequalities and some critical elliptic and parabolic problems. J. Differential Equations, 144, 441-476 (1998)

[G] Gelfand, I.M.: Some problems in the theory of quasilinear equations. Amer. Math. Soc. Transl., 29, 295-381 (1963)

[JL] Joseph, D.D., Lundgren, T.S.: Quasilinear Dirichlet problems driven by positive sources. Arch. Rat. Mech. Anal., 49, 241-269 (1973)

[KV] Kalton, N.J., Verbitsky, I.E.: Nonlinear equations and weighted norm inequalities. Trans. Amer. Math. Soc., 351, 3441-3497 (1999)

[Ma1] Martel, Y.: Uniqueness of weak extremal solutions of nonlinear elliptic problems. Houston J. Math., 23, 161-168 (1997)

[Ma2] Martel, Y.: Complete blow up and global behavior of solutions of $u_{t}$ $\triangle u=g(u)$. Ann. Inst. H. Poincare Anal. Non Lineaire., 15, 687-723 (1998)

[MP] Mignot, F., Puel, J.-P.: Sur une classe de problèmes non linéaires avec nonlinéarité positive, croissante, convexe. Comm. P.D.E., 5, 791-836 (1980)

[N1] Nedev, G.: Regularity of the extremal solution of semilinear elliptic equations. C. R. Acad. Sci. Paris Sér. I Math., 330, 997-1002 (2000)

[N2] Nedev, G.: Extremal solutions of semilinear elliptic equations. Preprint, 2001.

[PV] Peral, I., Vázquez, J.L.: On the stability or instability of the singular solution of the semilinear heat equation with exponential reaction term. Arch. Rat. Mech. Anal., 129, 201-224 (1995)

[V] Vázquez, J.L.: Domain of existence and blow-up for the exponential reaction-diffusion equation. Indiana Univ. Math. J., 48, 677-709 (1999)

[VZ] Vázquez, J.L., Zuazua, E.: The Hardy inequality and the asymptotic behaviour of the heat equation with an inverse-square potential. J. Funct. Anal., 173, 103-153 (2000) 